\title{
Patient-Reported Experiences in Outpatient Telehealth Heart Failure Management
}

\author{
Karanvir S. Raman a, d, John R. Vyselaar ${ }^{\mathrm{b}, \mathrm{c}}$
}

\begin{abstract}
Background: With the onset of coronavirus disease 2019 (COVID-19), the delivery of routine outpatient heart failure (HF) care abruptly shifted to telehealth. Appropriate HF management extensively relies upon patient-reported symptoms. With the growing attention towards patient-centered care, our team recognized an invaluable opportunity to solicit patient-reported subjective experiences regarding telehealth.
\end{abstract}

Methods: In total, 127 patients with a known diagnosis of HF were contacted by phone for participation in an online questionnaire. The tool consisted of questions generated by the investigators and from prior validated patient-reported experience measures. The intention was to assess the quality of care in our HF clinic and to solicit feedback regarding telehealth.

Results: Thirty-five patients provided a response. Questions with the most favorable outcomes were in line with our predetermined themes of interpersonal matter, communication, and perceived quality of care. The worst performing questions exhibited a lack of satisfaction with and perceived quality of telehealth. Only $9 \%(\mathrm{n}=3)$ preferred follow-up via telehealth, $69 \%(\mathrm{n}=22)$ preferred in-person, and $22 \%$ $(\mathrm{n}=7)$ were indifferent.

Conclusions: Given the multitude of benefits of telehealth, especially appropriate social distancing, telehealth is quite likely here to stay. In sum, with the rapid change in care delivery, patients currently perceive the care delivered via telehealth to be of inferior quality. This lack of quality can be largely attributed to the lack of physical examination, depersonalization of healthcare, and likely, a lack of familiarity with the platform. We urge our colleagues to solicit similar feedback from their patients to improve their own telehealth efforts.

Keywords: Heart failure; Telehealth; PREM; PROM

Manuscript submitted March 14, 2021, accepted May 1, 2021

Published online May 14, 2021

${ }^{a}$ Faculty of Medicine, The University of British Columbia, Vancouver, BC, Canada

bDivision of Cardiology, The University of British Columbia, Vancouver, BC, Canada

${ }^{c}$ Vancouver Coastal Health, Vancouver, BC, Canada

${ }^{\mathrm{d} C o r r e s p o n d i n g ~ A u t h o r: ~ K a r a n v i r ~ S . ~ R a m a n, ~ F a c u l t y ~ o f ~ M e d i c i n e, ~ T h e ~ U n i-~}$ versity of British Columbia, Vancouver, BC, Canada.

Email:karan.raman@outlook.com

doi: https://doi.org/10.14740/cr1253

\section{Introduction}

With the onset of coronavirus disease 2019 (COVID-19), pre-existing telehealth adoption trends have been accelerated out of necessity. In particular, there has been a broad shift of routine follow-up appointments to a virtual platform utilizing either phone or web calls. Given the lack of forethought and unexpected nature of the global pandemic, many care providers have resorted to a quick adoption approach, with little feedback and/or guidance to support their efforts.

In early March of 2020, as with the rest of the nation, our Heart Function Clinic had adopted a telehealth approach for routine follow-up of our heart failure (HF) patients. In HF, the disease process is naturally waxing and waning with multiple episodes of acute exacerbation often requiring hospitalization. Management consists of the utmost patient responsibility regarding education, diet, and medication compliance. Due to the intimate relationship of care provider and patient in HF care, the experiences and outcomes from a patient's perspective are at the forefront of adequate patient-centered management plans.

The literature regarding delivery of patient-centered care has highlighted the role of patient-reported outcome measures (PROMs) and patient-reported experience measures (PREMs). PROMs seek to measure the objective health outcomes of a patient over a longitudinal horizon, and can be generic or disease specific [1]. PREMs on the other hand, seek to measure a patient's subjective perspective regarding the healthcare they have received and the extent to which certain processes occur during the episode [2].

During the acute paradigm shift towards telehealth, our team recognized the opportunity to examine the quality of care delivered to HF patients virtually. We assessed both patientreported experiences regarding the telehealth delivery of routine HF care and procured baseline objective measures of their cardiac health.

\section{Materials and Methods}

\section{Survey tool}

The questionnaire utilized in our study consisted of three broad segments. The first segment was composed of the Kansas City Cardiomyopathy Questionnaire-12 (KCCQ-12) to establish sample baseline summary scores. The KCCQ-12 was selected due to its strong internal validity, prognostic power, and suc- 
cinct nature $[3,4]$. Segments 2 and 3 consisted of the PREMs. While no validated PREMs exist for HF, we built a tool with the help of the Vancouver Coastal Health Quality Improvement Department. Our team sourced ideas from prior validated tools such as the Consultation and Relational Empathy Measure, Patient Satisfaction Questionnaire Short-Form, Picker Patient Experience Questionnaire, and OutPatient Experiences Questionnaire, as these best matched our patient population [5-8]. The questions are intended to address the following domains of care: communication, interpersonal matter, financials, perceived time spent, perceived quality, satisfaction, technical issues, and preferences regarding follow-up communication medium. In particular, segment 2 included 24 questions regarding the extent of agreement among various indicators of care quality in our HF clinic and virtual care. Segment 3 consisted of 1 - 2 questions, and was tailored to procure the different reasons for their preferred follow-up communication medium (i.e., inperson, virtual, or indifferent).

\section{Data handling and analysis}

Questionnaire execution utilized the Qualtrics platform for initial data gathering and was distributed via patient-provided email. Final results were subsequently translated into Microsoft Excel 2019 for analysis and chart construction. To standardize and facilitate comparison, the variable Likert scales are presented as percentage of total responses in the "Top box" option. Continuous quantitative variables are presented as mean \pm standard deviation. Thematic analysis for qualitative data was conducted by one of the investigators. Themes were developed via inductive reasoning after viewing the data and categorizing themes as they appeared. The questionnaire and definition of "Top box" responses may be found here (Supplementary Material 1, www.cardiologyres.org).

\section{Population}

We evaluated the responses from patients followed in the Heart Function Clinic at the North Shore Heart Centre in North Vancouver, BC, Canada. Patients included in this study had a known diagnosis of HF, were currently living at home independently, and were seen at least once from March 13, 2020 to August 15, 2020 via telehealth. Patients were recruited on a rolling basis from July 15, 2020 to August 20, 2020 from 9:00 am to 7:00 pm via telephone for participation. To improve recruitment efforts, patients who did not answer the phone call were contacted two additional times and were provided with one email reminder for not completing their questionnaire. The care providers included three Canadian board-certified cardiologists and one nurse practitioner with HF expertise.

\section{Ethics approval}

Institutional review board approval was not applicable, as the original intent of the study was quality improvement in a local outpatient cardiology clinic. As such, review board and/or ethics approval was not indicated as per the University of British Columbia's policy. The secondary intent to publish our findings was decided after procurement of the results presented in our study. Ethics approval was not required according to the University of British Columbia Office of Research Ethics.

\section{Results}

\section{All participants}

A total of 127 individuals were originally contacted, 61 were interested in participating, 35 provided responses, and of which, 24 completed the questionnaire in its entirety. Of the 35 respondents, 23 were male and 12 were female. A baseline KCCQ-12 summary score was obtained to facilitate comparison with subsequent iterations. Summary score for our 35 participants was $46.77 \pm 12.52$ out of 70 total points.

A summary of results may be found in Table 1. The three questions with the highest percentage of "Top box" responses were as follows: $85 \%$ of respondents agreed that the care team really cared about them as a person; an additional $81 \%$ suggest that the care team both showed respect for what they had to say and demonstrated adequate preparation regarding their medical history; $78 \%$ of respondents suggested that their care team always listened to them carefully. The "Top box" responses clearly fall into our predetermined themes of interpersonal matter, communication, and perceived quality of care.

On the other hand, the bottom four worse performing questions, with the least amount of "Top box" responses were as follows. Only $38 \%$ rated their experience with virtual HF care a 9 or 10 , with 10 being the best possible experience. While not listed in Table 1, the middle box response of 7 to 8 , garnished another $46 \%$ of total responses. Only $25 \%$ of respondents were "completely confident" in the care team providing an appropriate management plan through virtual appointments. Ironically, only $15 \%$ of respondents suggested that their care team discussed the financial impact of their care over the last 3 months. Lastly, only 12\% reported the quality of virtual appointments as being "much better" than their previous in-person appointments and 46\% suggested it was "somewhat worse than in-person". The dominant themes revolve around perceived quality and satisfaction in telehealth HF care.

Going forward, only $9 \%(\mathrm{n}=3)$ of participants would prefer follow-up via telehealth, $69 \%(\mathrm{n}=22)$ prefer the conventional in-person approach, and $22 \%(\mathrm{n}=7)$ were indifferent to either option.

\section{Indifferent cohort}

The seven patients who were indifferent to telehealth or in-person follow-up had a mean age of $81.2 \pm 10.2$ years. Regarding virtual appointments, $57 \%$ of the indifferent cohort noted that telehealth offers scheduling flexibility and superior travel time to be the primary decision drivers. Interestingly, none suggested that perceived quality of care to be a reason. When asked 
Table 1. Questionnaire "Top box" Responses

“Top box" responses by survey questions $\quad$ N $\quad$ \%

In the last 3 months, how often did your physician or nurse practitioner...

Listen to you carefully?

$\begin{array}{ll}25 & 78 \% \\ 26 & 81 \% \\ 16 & 50 \% \\ 26 & 81 \% \\ 18 & 56 \% \\ 24 & 75 \%\end{array}$

Show respect for what you had to say?

Spend enough time with you?

Seem to know the important information about your medical history?

Encourage you to ask questions?

How often did your physician and/or nurse practitioner explain the following things in a way that was easy to understand?

Your test results and prognosis

$22 \quad 67 \%$

The reason for tests, medications, and treatments

$2370 \%$

The possible side effects of your medications

$13 \quad 39 \%$

Do you feel that your physician and/or nurse practitioner really care about you as a person?

$29 \quad 85 \%$

In the last 3 months, were you ever worried or concerned about the financial impact of the medical care that you need?

$24 \quad 71 \%$

In the last 3 months, did you and your physician and/or nurse practitioner talk

$5 \quad 15 \%$

about the financial impact of the medical care that you need?

How often was your virtual appointment well organized?

$15 \quad 52 \%$

Compared to your in-person appointment (pre-COVID-19), how would you

$312 \%$

rate the quality of the virtual appointments you attended?

How confident do you feel that your physician and/or nurse practitioner can provide

7 an appropriate management plan through virtual appointments?

Did the North Shore Heart Center give you enough information about how to participate in the virtual appointments?

Overall, how easy or difficult was it for you to participate in your virtual appointments?

Using any number from 0 to 10 , where 0 is the worst experience possible and 10 is the best experience possible, overall, how would you rate the experience with virtual appointments for your heart failure?

Did you have any problems during your virtual appointments...? Please select all the apply.

No, I did not have any problems.

Yes, I had problems with sound quality.

$26 \%$

Yes, I had problems with video quality.

$13 \%$

Yes, I had problems with the connection (such as poor cell service or internet connection).

$13 \%$

Yes, I am not familiar with the technology.

$13 \%$

Yes, I was concerned for the privacy of my health information.

Other (please specify):

$0 \%$

Would you prefer your future appointments to be virtual or in-person?

Virtual

$11 \quad 34 \%$

In-person

Either

\begin{tabular}{ll}
3 & $9 \%$ \\
\hline 22 & $69 \%$ \\
7 & $22 \%$ \\
\hline
\end{tabular}

Patients that prefer either: mean age \pm SD $(81.2 \pm 10.2)$

Please describe why you prefer virtual appointments. Please select all that may apply.

\begin{tabular}{lc} 
Quality of care & $0 \%$ \\
Travel time & 4 \\
Cost & $57 \%$ \\
Comfort & $29 \%$ \\
Scheduling flexibility & 3 \\
Physical access (transportation, stairs, wheelchair, etc.) & $43 \%$ \\
\hline
\end{tabular}


Table 1. Questionnaire "Top box" Responses - (continued)

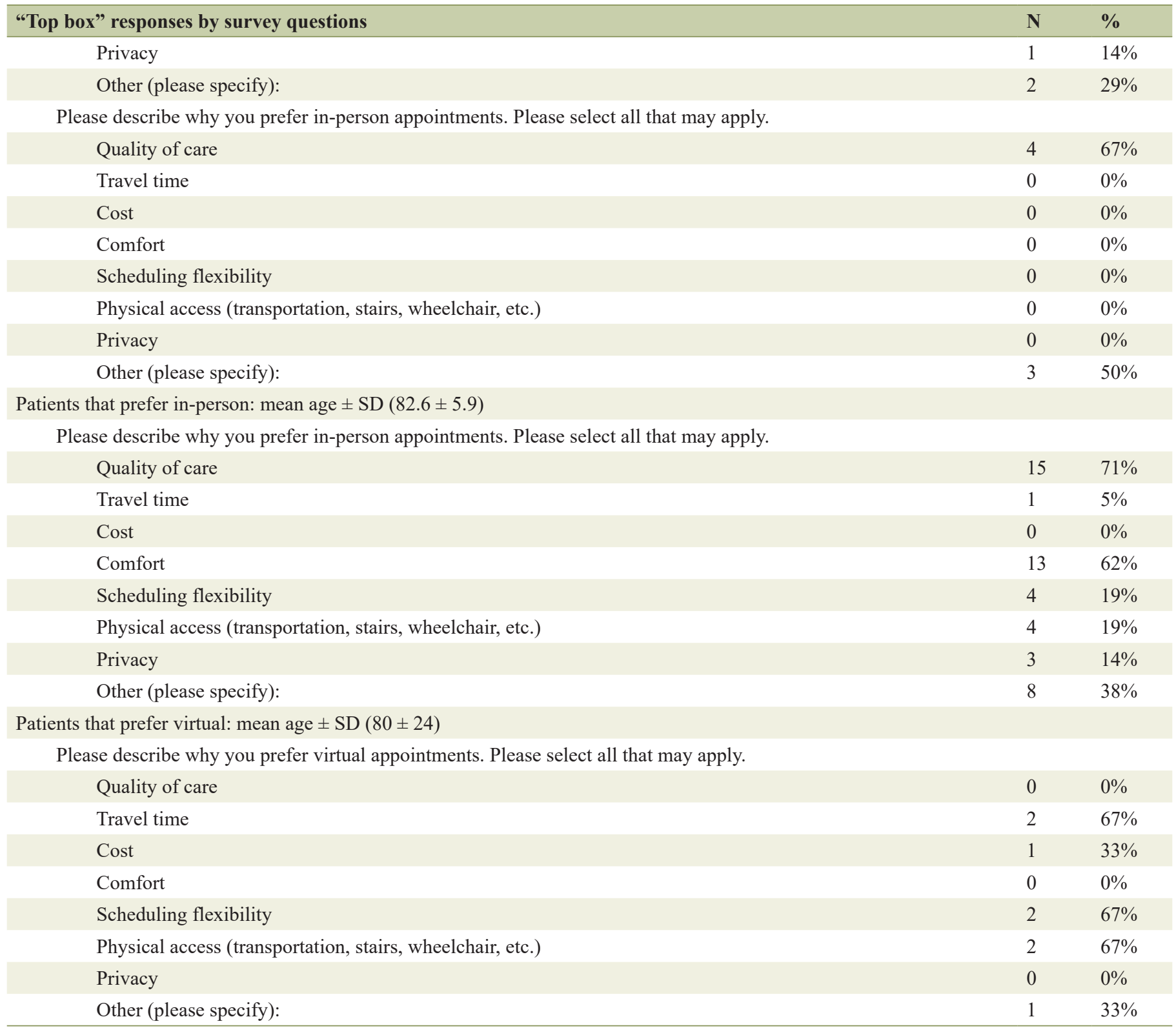

COVID-19: coronavirus disease 2019; SD: standard deviation.

about their thoughts regarding in-person appointments, 67\% noted that perceived quality of care as the reason. Relevant subjective responses included perceived value from physical examination absent in telehealth.

\section{In-person cohort}

Of the 22 patients that preferred in-person follow-up, mean age was $82.6 \pm 5.9$ years. Likewise, $71 \%$ of the cohort noted quality of care to be a notable factor, with $62 \%$ also highlighting the added comfort from in-person appointments. Thirtyeight percent of respondents provided their subjective opinion including a lack of personal connection inherent to telehealth: "Personal interaction is so hard to come by right now, it is very appreciated" and "Prefer to speak with a doctor in person as virtual calls feel impersonal". Once again, perceived quality of care extends beyond the objective disease process such as: "The doctor is able to make a more complete evaluation of the patient under the latest observations, i.e., evaluation of the patient's condition not the significance of some indicators".

\section{Virtual cohort}

Of the three patients that preferred virtual follow-up, they had 
a mean age of $80.0 \pm 24$ years. The primary factors influencing this decision included $67 \%$ of respondents mentioning travel time, scheduling flexibility, and physical access. One patient mentioned the value of social distancing as their subjective response. It should be noted that the total number of respondents in this cohort was only three, limiting our ability to draw significant conclusions.

\section{Final comments}

Patients were provided a final opportunity to mention any concerns or comments not yet discussed. Two patients provided positive concluding comments regarding their care such as "Very satisfied" and "Doing an excellent job as is". A single patient reiterated the reassurance and value from personal connection with a health expert given the uncertain nature of HF: "That is why personal contact is so important, sometimes you can get help wrestling with the great imponderables. You have experience; I do not." Lastly, a lack of perceived quality of care was mentioned: "The doctor makes a more complete and personal evaluation possible by in-person meetings"; with physical examination playing a significant role: "I miss getting blood pressure checks and heart monitoring" and "Unable to take an electrocardiogram (ECG) or blood pressure by virtual meetings".

\section{Discussion}

Inglis et al conducted a meta-analysis of randomized controlled trials (RCTs) exploring the efficacy of structured telephone support or telemonitoring for HF patients and compared the results to the standard practice of in-person appointments [9]. The results illustrate the utility of telehealth, as the approach reduced all-cause mortality and HF-related hospitalizations. Of note, the Tele-HF study analyzed by Chaudhry et al indicated a non-inferiority of telehealth when judging for readmission for any reason or death within 180 days when compared to the standard of practice [10].

With the ever increasing rate of technological improvement, certainly a number of advantages now exist with the utilization of telehealth. Namely, telehealth offers the opportunity to provide care to underserved or inaccessible areas, temper the ballooning costs of our healthcare system, logistical flexibility, and added comfort [11-14]. For the management of HF, patient education and compliance with their medication regimen is of the utmost importance, both of which can be augmented via telehealth $[9,15,16]$. Beyond the clinical significance of telehealth, patient satisfaction may also be maximized with a virtual approach; in fact patients may eventually prefer the novel medium.

According to Buvik et al, patients requiring orthopedic surgery consults noted no difference in satisfaction and a significant proportion opted for video-assisted remote consults for follow-up [17]. In a comparable population of HF patients, for implantable cardioverter defibrillator (ICD) monitoring, the results of a 2-year study by Timmermans et al demonstrat- ed that $43 \%$ of patients preferred remote monitoring, $19 \%$ in clinic, and 38\% had no preference [13]. Perhaps longitudinal exposure revealed the benefits of and fostered comfort with said platform, providing hope for our recently adopted change and lackluster results from a preliminary assessment. While the upside of telehealth over a longitudinal horizon demonstrates favorable outcomes, to the best of our knowledge, there exist few studies exploring HF patients' subjective perspectives towards telehealth. Subjective accounts to assist practitioners are especially lacking during this acute global pandemic which imposed swift and radical change in the delivery of HF care.

In our study, after the initial telehealth appointment, the preference for the standard in-person follow-up was the clear favorite with $69 \%$ of responses; while another $22 \%$ were indifferent, only $9 \%$ opted for continued telehealth follow-up. Investigating this finding reveals the apparent underlying driver to be a lack of perceived quality in telehealth. While this driver is quite ambiguous, delving into the accompanying results may suggest that this lack of quality can be attributed to a handful of recurrent themes; for instance, the missing physical examination. While routine HF care largely relies on subjective symptoms such as dyspnea and fatigue for pharmacotherapy modification, the care model patients are accustomed to do not fit the telehealth model. By repeat association, perhaps patients are taught the importance and expectation of physical examination; ultimately owing to a lack of education regarding best practices in disease management. Therefore, clinicians should take the time to reiterate that the telehealth appointment will be just as exhaustive and reassure patients that testing and/or in-person appointments will be offered if indicated [18].

Not to mention the emergency contextual environment in which our telehealth strategy was adopted. It is safe to assume that nearly all patients are largely unfamiliar to such an approach to medicine, contributing to the lack of perceived quality. This was of course compounded by the fact that our cohort's mean age was greater than 80 years and as such, have most likely grown accustomed to and comfortable with the conventional appointments over the course of their long lives. Therefore, it is understandable that given this lack of trust in telehealth and an overlaying extensive disease burden, our patients would be apprehensive to this novel care delivery medium and consequently, judge it to be of inferior quality. In response, clinicians should take the time to inform their patients of the benefits, functions, and limitations with appropriate mitigation plans; a thorough discussion is likely to increase buy-in for electronically delivered services [19].

Lastly, patient wellness and perception of quality certainly include the interpersonal component of the physician-patient relationship [20]. The recurrent emphasis in our questionnaire on the lack of emotional connection and depersonalization of medicine via telehealth surely needs addressing. This has gone so far as patients suggesting that "Personal interaction is so hard to come by", and have expressed a desire in "getting to know the doctor". Knowing the therapeutic power of effective interpersonal communication, we must take steps to not only treat the disease, but the patient as a whole with compassion while maintaining appropriate social distance. More often than not, similar communication techniques such as building social rapport, summarizing, checking understanding, and extending 
opportunity to ask questions used in face to face meetings can be applied to telehealth to yield similar results [18].

While our results exhibit limited support for telehealth in $\mathrm{HF}$, it is encouraging to note that this is a preliminary result from a hastily executed strategy. Regardless of the extensive age of HF patients, providers should be confident that with enough time and education, patients are willing to adapt to and accept telehealth [21]. Ultimately, our findings while highlighting our pitfalls, also offer actionable targets for improvement going forward.

\section{Limitations}

A number of limitations exist. First, our study was limited by a lower-than-expected participation rate. Only 35 of the 127 contacted participants provided a response; further, only 24 completed our questionnaire in its entirety. Accordingly, the results may not be an adequate representation of the population at large, limiting the predictive value of our study. Next, our questionnaire utilized both email and Qualtrics for distribution and data collection. This introduces an inherent bias towards technologically inclined participants which may skew our results towards virtual preferences. Although, we had only three patients prefer virtual appointments for follow-up. To increase participation, participants were allowed to complete the survey partially, leaving us with incomplete questionnaires. The questionnaire was conducted from the Heart Function Clinic following our agreed upon best practices. These practices may or may not represent the preferences of other cardiologists. Additionally, there is no delineation in results from care delivered by a HF nurse practitioner or cardiologist. Lastly, data were not stratified by distance to clinic, limiting our ability to elucidate the effect of travel time as a decisive factor.

\section{Conclusions}

It is no doubt that changing decades-long practice will encounter significant pushback from a population who is apprehensive to acute change. However, given the multitude of benefits from telehealth particularly, during a pandemic and non-inferiority, it is a difficult change we must pursue in stride. Due to the intimate relationship between provider and patient in $\mathrm{HF}$, patients tend to be a treasure trove of valuable information. In sum, we believe that patients currently perceive the care delivered via telehealth to be of inferior quality due reasons including a lack of physical examination, emotional detachment from care providers, and general unfamiliarity. A number of changes will be employed in our telehealth clinic to address these shortcomings; and we hope that subsequent iterations of our questionnaire can provide valuable insights. Likewise, we urge our colleagues to solicit similar feedback from their patients to not only sustain, but improve the delivery of HF care via telehealth.

\section{Supplementary Material}

Suppl 1. Questionnaire and definition of "Top box" responses.

\section{Acknowledgments}

We would like to acknowledge both Allison Chiu and Serena Bertoli-Haley from the Vancouver Coastal Health Quality Improvement Department for assisting in the creation of the questionnaire and providing feedback on the virtual execution strategy.

\section{Financial Disclosure}

No source was obtained.

\section{Conflict of Interest}

None to declare.

\section{Informed Consent}

Not applicable.

\section{Author Contributions}

Karanvir S. Raman: study design, study execution (creation of database/questionnaire, patient recruitment, and collection of data), and primary author of the manuscript (wrote the paper). John R. Vyselaar: study design (original idea), study execution (guidance on database/questionnaire creation and primary cardiologist for the patients included in the study), and secondary author of the manuscript (revised draft versions).

\section{Data Availability}

The data underlying this article will be shared on reasonable request to the corresponding author.

\section{References}

1. Black N. Patient reported outcome measures could help transform healthcare. BMJ. 2013;346:f167.

2. Male L, Noble A, Atkinson J, Marson T. Measuring patient experience: a systematic review to evaluate psychometric properties of patient reported experience measures (PREMs) for emergency care service provision. Int J Qual Health Care. 2017;29(3):314-326.

3. Spertus JA, Jones PG. Development and validation of a short version of the Kansas City cardiomyopathy questionnaire. Circ Cardiovasc Qual Outcomes. 2015;8(5):469-476.

4. Yee D, Novak E, Platts A, Nassif ME, LaRue SJ, Vader JM. Comparison of the Kansas City cardiomyopathy questionnaire and minnesota living with heart failure questionnaire in predicting heart failure outcomes. Am J Cardiol. 2019; 
123(5):807-812.

5. Jenkinson C, Coulter A, Bruster S. The Picker Patient Experience Questionnaire: development and validation using data from in-patient surveys in five countries. Int J Qual Health Care. 2002;14(5):353-358.

6. Mercer SW, Maxwell M, Heaney D, Watt GC. The consultation and relational empathy (CARE) measure: development and preliminary validation and reliability of an empathy-based consultation process measure. Fam Pract. 2004;21(6):699-705.

7. Garratt AM, Bjaertnes OA, Krogstad U, Gulbrandsen P. The OutPatient Experiences Questionnaire (OPEQ): data quality, reliability, and validity in patients attending 52 Norwegian hospitals. Qual Saf Health Care. 2005;14(6):433437.

8. Thayaparan AJ, Mahdi E. The Patient Satisfaction Questionnaire Short Form (PSQ-18) as an adaptable, reliable, and validated tool for use in various settings. Med Educ Online. 2013;18:21747.

9. Inglis SC, Clark RA, Dierckx R, Prieto-Merino D, Cleland JG. Structured telephone support or non-invasive telemonitoring for patients with heart failure. Cochrane Database Syst Rev. 2015;10:CD007228.

10. Chaudhry SI, Mattera JA, Curtis JP, Spertus JA, Herrin J, Lin Z, Phillips CO, et al. Telemonitoring in patients with heart failure. N Engl J Med. 2010;363(24):2301-2309.

11. Comin-Colet J, Enjuanes C, Verdu-Rotellar JM, Linas A, Ruiz-Rodriguez P, Gonzalez-Robledo G, Farre N, et al. Impact on clinical events and healthcare costs of adding telemedicine to multidisciplinary disease management programmes for heart failure: Results of a randomized controlled trial. J Telemed Telecare. 2016;22(5):282-295.

12. Kane-Gill SL, Rincon F. Expansion of Telemedicine Services: Telepharmacy, Telestroke, Teledialysis, Tele-Emergency Medicine. Crit Care Clin. 2019;35(3):519-533.

13. Timmermans I, Meine M, Szendey I, Aring J, Romero Roldan J, van Erven L, Kahlert P, et al. Remote moni- toring of implantable cardioverter defibrillators: Patient experiences and preferences for follow-up. Pacing Clin Electrophysiol. 2019;42(2):120-129.

14. Krum H, Forbes A, Yallop J, Driscoll A, Croucher J, Chan $\mathrm{B}$, Clark R, et al. Telephone support to rural and remote patients with heart failure: the Chronic Heart Failure Assessment by Telephone (CHAT) study. Cardiovasc Ther. 2013;31(4):230-237.

15. Antonicelli R, Mazzanti I, Abbatecola AM, Parati G. Impact of home patient telemonitoring on use of beta-blockers in congestive heart failure. Drugs Aging. 2010;27(10):801805.

16. Antonicelli R, Testarmata P, Spazzafumo L, Gagliardi C, Bilo G, Valentini M, Olivieri F, et al. Impact of telemonitoring at home on the management of elderly patients with congestive heart failure. J Telemed Telecare. 2008;14(6):300-305.

17. Buvik A, Bugge E, Knutsen G, Smabrekke A, Wilsgaard T. Patient reported outcomes with remote orthopaedic consultations by telemedicine: A randomised controlled trial. J Telemed Telecare. 2019;25(8):451-459.

18. Sabesan S, Allen D, Caldwell P, Loh PK, Mozer R, Komesaroff PA, Talman P, et al. Practical aspects of telehealth: doctor-patient relationship and communication. Intern Med J. 2014;44(1):101-103.

19. Ottenberg AL, Swetz KM, Mueller LA, Gerhardson S, Mueller PS. "We as Human Beings Get Farther and Farther Apart": the experiences of patients with remote monitoring systems. Heart Lung. 2013;42(5):313-319.

20. Toh N, Pawlovich J, Grzybowski S. Telehealth and patient-doctor relationships in rural and remote communities. Can Fam Physician. 2016;62(12):961-963.

21. Clark RA, Yallop JJ, Piterman L, Croucher J, Tonkin A, Stewart S, Krum H, et al. Adherence, adaptation and acceptance of elderly chronic heart failure patients to receiving healthcare via telephone-monitoring. Eur J Heart Fail. 2007;9(11):1104-1111. 